\title{
Biological and Therapeutic Applications of Small RNAs
}

\author{
Kun Cheng • Ram I. Mahato
}

Received: 12 October 2011 / Accepted: 12 October 2011 / Published online: 28 October 2011

(C) Springer Science+Business Media, LLG 2011

In the past decade, small RNAs including small interfering RNA (siRNA) and microRNA (miRNA) have attracted intense interest due to their unique roles in fundamental biological processes by regulating the stabilities and functions of mRNAs. As illustrated in Fig. 1, the numbers of publications related to siRNA and miRNA have increased dramatically in recent years. Although miRNA was discovered earlier than siRNA, its importance was not fully realized as siRNA from 2000 to 2005 . However, while the siRNA publications reached a plateau after 2007, the number of publications on miRNA has increased exponentially since 2005 .

siRNAs are artificial double-stranded RNAs of 21-23 nt in length, while miRNAs are endogenous singlestranded RNAs of 21-25 nt. Both siRNA and miRNA are non-coding RNAs that post-transcriptionally suppress gene expressions by binding to their complementary mRNAs. The major difference between siRNA and miRNA is that each siRNA only specifically targets a single mRNA and induces its degradation, while a single miRNA targets hundreds of mRNAs either by blocking protein translation or destabilizing the mRNA molecules. This difference comes from the fact that siRNA

\section{K. Cheng $(\bowtie)$}

Division of Pharmaceutical Sciences, School of Pharmacy

University of Missouri-Kansas City

2464 Charlotte Street, HSB 5250

Kansas City, Missouri 64108-27I8, USA

e-mail: chengkun@umkc.edu

\section{R. I. Mahato $(\bowtie)$}

Department of Pharmaceutical Sciences

University of Tennessee Health Science Center

19 S Manassas, RM 224

Memphis, Tennessee 38103-3308, USA

e-mail: rmahato@uthsc.edu always forms a perfect match to its complementary mRNA, while miRNA only forms imperfect matches to its multiple mRNA targets (1-3).

This theme issue focuses on recent advances in the biological and therapeutic applications of siRNA and miRNA. There are six articles in this issue highlighting research from studying gene functions to delivery to therapeutic application of siRNA. Since its discovery, siRNA has been widely applied as a powerful tool to study genetic functions of a specific gene. To further expand the applications of siRNA in gene function studies, Friedman et al. describe a novel light-activated RNA interference (RNAi) strategy by incorporating photolabile groups (di-methoxy nitro phenyl ethyl or DMNPE) to multiple double-stranded precursors of siRNA (dsRNA). Phosphorothioate linkages are also incorporated to increase the serum stability of the dsRNA. This strategy allows the control of the spacing, timing, and amount of gene expressions by adjusting light exposure. A successful light-controllable RNAi is particularly useful for the study of developmental biology or targeted delivery to specific sites in the body.

Another promising application of siRNA is the treatment of various diseases by silencing their key modulators. Currently, there are 13 ongoing clinical trials using siRNA for numerous diseases. A review by Schaffer et al. discusses basic action mechanisms and therapeutic applications of RNAi in antiviral therapy. Design considerations, delivery strategies, computational insight, and areas for future improvement are described. Another article by Nemunaitis and colleagues summarizes the potentials, challenges, and current status of small RNA-based cancer therapies. As an example of developing siRNA therapy, Cheng's group explore the potential therapeutic application of PCBP2 siRNA in treating alcoholic liver fibrosis which is charac- 
Fig. I PubMed search of publications related to "RNA interference" and "miRNA/miroRNA" for each year. The numbers in $201 \mathrm{I}$ are projected based on the number of publications from the first 9 months.

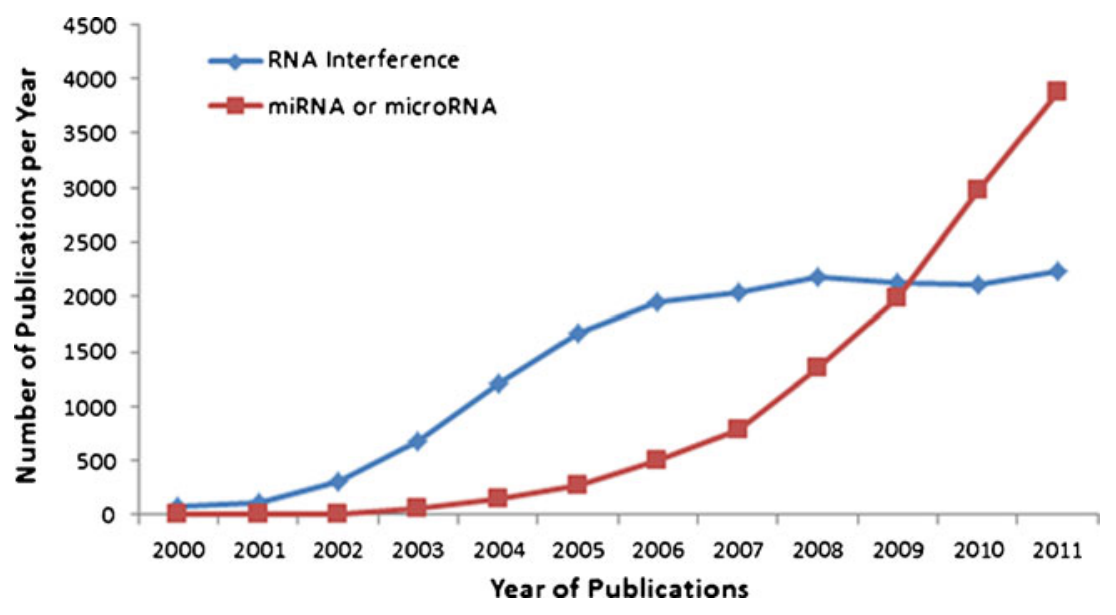

terized with accumulated type I collagen. The accumulation of type I collagen in fibrotic liver is primarily due to an increase in the half-life of its mRNA, which is stabilized by $\alpha$-complex protein-2 ( $\alpha \mathrm{CP} 2)$, encoded by the PCBP2 gene. Therefore, the study aims to reduce the half-life of the type I collagen mRNA by suppressing the PCBP2 gene using siRNA. They identify a potent PCBP2 siRNA to silence the PCBP2 expression, which subsequently increases the decay rate of the type I collagen mRNA, offering a potential treatment for various fibrosis diseases, including liver fibrosis. The greatest challenge in achieving siRNA therapy is the efficient delivery of siRNA to target cells. This is reflected in the fact that most of the siRNAs in clinical trials are designed for local rather than systemic administration. A review by Singh et al. summarizes the challenges and recent progress in overcoming various biological barriers in the systemic delivery of small RNAs to their target cells. Cellular uptake, endosomal release, and intracellular transport of small RNAs are discussed. Off-target effect caused by non-specific delivery is also discussed in this article. Oh and colleagues have developed a strategy to successfully co-deliver siRNA and a chemical MEK inhibitor to target tumor cells using cationic liposomes. The MEK inhibitor is first encapsulated in the lipid layers of the N',N"-dioleylglutamide-based cationic liposomes, and then the siRNA is complexed with the liposomes.

It is estimated that there are over 900 miRNAs in mammalian cells and that approximately $30 \%$ of all genes are regulated by miRNA. In spite of the accumulation of a large number of validated miRNAs, predicting gene targets of miRNAs remains a big challenge because a single miRNA may target as many as several hundred genes. Hence, a number of computational algorithms have been designed to predict miRNA gene targets, which is critical for predicting potential side effects and toxicity profiles associated with miRNA-based therapeutics (4). For example, Tan et al. describe a domain-specific knowledge base built upon the Ontology for MicroRNA Targets (OMIT) to facilitate the prediction of miRNA target genes. However, it is worth noting that only experimental evidence can eventually validate these targets.

There has been an explosion of interest in miRNAs as they can be exploited as biomarkers as well as therapeutic targets in a variety of diseases. The review article by Kerin $e t$ al. outlines the underlying rationale for considering miRNAs as therapeutic targets or agents. Potential challenges in achieving a successful miRNA therapy are summarized in this article. The authors also highlight the promise of regulating miRNA for treating different diseases. Among them, cancer therapy is the most important application of miRNA. This is also evidenced by the fact that nearly three-quarters of the miRNA publications are associated with various cancers. Therefore, this theme issue presents four articles focused on the application of miRNA in cancer therapy.

As Jeang and Schmittgen summarize in their review articles, aberrant miRNA expressions are always associated with various cancers. Some miRNAs (oncogenic miRNAs) are overexpressed in tumors, while others (tumor suppressor miRNAs) are expressed at reduced levels. Oncogenic miRNA levels can be suppressed by several RNAi-type strategies such as anti-miRNA oligonucleotides, miRNA sponges, and miRNA masking. Conversely, reduced miRNA levels can be restored by three approaches: direct delivery of miRNA mimics, introduction of miRNA gene via DNA vector, or small molecules that can reverse the epigenetic changes caused by miRNA.

This theme issue also includes several research articles in the exploration of miRNA for cancer therapy. The article from Batchu reveals a reduced miR-101 expression in epithelial ovarian carcinoma cells in comparison to normal cells. Delivery of miR-101 into the cells suppresses cell proliferation and invasiveness of epithelial ovarian carcinoma cells in vitro and decreases tumor growth in a xenograft model. miRNA has also been 
found associated with the regulation of multidrug resistance (MDR) in cancer cells. Shim and colleagues demonstrate that miR-19 promotes MDR of breast cancer cells to chemotherapy drugs. Accordingly, inhibition of miR-19 restores sensitivity of the cells to various chemotherapy agents, including Taxol, VP-16, and mitoxantrone. This study suggests that miRNA can be a valid target to prevent drug resistance in tumor cells.

In summary, small RNAs have been quite successful in biological application as a tool to study gene functions. Despite rapid progress, therapeutic application of small RNAs is still in its infancy. Off-target effects and lack of efficient delivery remain the primary challenges for small RNA-based therapy. Indeed, these challenges have plagued both industrial and academic research. This coupled with the recent failures in several clinical trials has resulted in the stagnation of the field and dampened investor confidence. For successful translation to the clinic, breakthroughs in developing an efficient delivery system as well as reducing off-target effects are required, and this is where this theme issue can play a role in advancing the field. Therefore, we would like to express our sincere appreciation to all authors of this theme issue for their outstanding contributions. We also thank Dr. Saurabh Singh for offering useful suggestions and reviewing this editorial. We further hope this theme issue will stimulate further discussions leading to innovative approaches that may help to turn these fascinating small RNA molecules into therapeutics.

\section{INTERVIEW WITH DRS. KUN CHENG \& RAM I. MAHATO}

\author{
What do you think holds the key to your success \\ as a pharmaceutical scientist?
}

Cheng: Curiosity about the unknown and how things work is the key to my professional development. When I was a child, my most favorite book was Hundred Thousand Whys. Curiosity has always been a driving force in my life and motivates me all the time to explore the unknown. Consequently, I have a broad range of interests, which have certainly helped me to be a scientist. Moreover, I love what I am doing as a pharmaceutical scientist and an educator. As Steve Jobs says, "You've got to find what you love.... If you haven't found it yet, keep looking. Don't settle." Luckily, I have found it.

Mahato: Perseverance and my interest in multidisciplinary research have contributed to my success. I also attribute my success to my students and post-doctoral fellows who have contributed new ideas and have opened novel research areas for my lab. They have helped my quest for constant learning and improvement.
What do you consider to be your key research accomplishments?

Cheng: We have explored various therapeutic applications of siRNA in the treatment of breast cancer, prostate cancer, and alcoholic liver fibrosis. We have identified several siRNAs that showed promising therapeutic potentials. We have developed numerous strategies to modify nucleic acids with different targeting moieties, which not only improve the stability of siRNA but also increase the cellular uptake by specific cells.

We have developed a peptide modification strategy to increase the solubility and specificity of a potential chemotherapy drug for prostate cancer. Cellular uptake of the peptide-drug conjugate is significantly improved in prostate cancer cells. This study provides an evidence-based conceptual strategy for development of other chemotherapy drugs with poor solubility and targetability.

We have also employed the phage display technology to identify peptide ligands for specific cells or receptors. We have shown that the newly identified peptide ligand specifically delivers a proapoptotic peptide to induce cell death in target cells, but not in other cells, indicating its promising potential for targeted drug delivery systems as well as imaging.

Mahato: My research has applied and developed innovative approaches to solve complex medical problems with small molecule and nucleic-acid-based therapeutics. We have worked to improve the design of gene delivery and expression systems and factors influencing their biodistribution and gene expression/silencing. We have explored the role of genetic modification of human islets for improved transplantation, and site specific delivery of oligonucleotides and siRNA for treating liver fibrosis, prostate and pancreatic cancer. We are now focusing on mesenchymal stem cells as therapeutic gene carriers and cancer stem cells as therapeutic targets.

\section{What was the turning point in your career?}

Cheng: The turning point in my career was joining Dr. Mahato's laboratory as a graduate student at the University of Tennessee Health Science Center and working in the emerging field of nucleic acid. Excellent training from Dr. Mahato's laboratory resulted in an exponential increase in my knowledge and experience in nucleic acid drug delivery, which is my current major research interest. In addition, Dr. Mahato provided me the opportunity and freedom to independently design research projects, which have greatly broadened my understanding of those areas and shaped my career as an independent scientist.

Mahato: I have had a number of crucial stages in my career, and many people are responsible for my success. Among them, the most memorable were my interactions with Drs. 
Vincent Lee and Mitsuru Hashida, which allowed my career as an independent scientist to take off.

\section{Which individuals most influenced your research career?}

Cheng: The first individual who influenced my research career is my grandfather, who is a physics professor at Nanjing University, one of the top universities in China. He inspired my first interest in science. To be a scientist like him has been a dream ever since I was a child. My three graduate advisors, Dr. Jiabi Zhu at Chinese Pharmaceutical University, Dr. Leeyong Lim at the National University of Singapore, and Dr. Ram I. Mahato at the University of Tennessee Health Science Center, are other individuals who most influenced my research career. They not only delivered their best knowledge to me, but also inspired my interest in pharmaceutical sciences and academia.

Mahato: I have been fortunate to work with world leaders like Dr. Vincent H.L. Lee, Sung Wan Kim, Mitsuru Hashida, among others. As a result, I have been able to take a multidisciplinary approach to my research. They were also instrumental in exposing me to many distinguished scientists working on different aspects of drug/gene delivery and material sciences.

\section{What is the key to developing successful collaborative relationships?}

Cheng \& Mahato: First, find people who can compensate for your weaknesses in a specific field. Second, be sincere to your collaborators. We should not expect that our collaborators will always agree with us. Candid communication and willingness to accept criticism are therefore critical to maintain a successful collaborative relationship.

\section{What is your philosophy of educating graduate students?}

Cheng: First of all, we need to inspire graduate students' interest in pharmaceutical sciences because I strongly believe that interest is the best teacher. While we deliver our best knowledge to graduate students, we should always help them develop the skills to think on their own. There is an old Chinese proverb, "to teach fishing is better than to give fish." The independent thinking ability allows graduate students to keep pace with the fast growing fields after their graduation. Moreover, writing and communication skills are also critical to their future success and growth.

Mahato: Pharmaceutical science students need to gain indepth knowledge of subjects like biochemistry, molecular biology, and organic and polymer chemistry. This helps them to develop original ideas and apply them in drug delivery sciences. In addition, students should be nurtured to become independent thinkers. They also need to develop their writing and communication skills.

What is the place for collaboration with industry in academia?

Cheng \& Mahato: Collaboration between industry and academia is crucial to the success of pharmaceutical sciences, especially in the current economic climate. It becomes more and more difficult for academic scientists to secure the shrinking federal funding. Collaboration with industry, such as contract research projects, provides an alternative pathway for academic scientists to support their research. On the other hand, it is also beneficial to the pharmaceutical industry because they do not need to hire permanent full-time employees for short-term research projects. Overall, this is a winning situation for both academia and industry.

\section{Pharmaceutical ccientists are faced with the dilemma of having} to publish in biomedical or basic science journals. does this mean cutting-edge science will not likely be featured in journals like Pharmaceutical Research?

Cheng \& Mahato: We believe that cutting-edge science in pharmaceutical sciences will still be featured in Pharmaceutical Research. As long as Pharmaceutical Research continues publishing high-quality manuscripts and maintains a high impact factor, scientists will be willing to publish their cutting-edge work here. Theme issues are an excellent strategy to summarize an emerging and challenging field in pharmaceutical sciences. A successful theme issue will draw the utmost attention of scientific community globally and stimulate further discussions that may lead to cutting-edge findings in those areas. That said, we as a journal have an audience comprised of pharmaceutical scientists working in academia, industry as well in regulatory affairs. Thus, we have to maintain a delicate balance when choosing articles for publication.

Where is the field of biological and therapeutic applications of small RNAs going? How do the articles in this theme section fill the gap?

Cheng \& Mahato: As mentioned in the editorial, the application of small RNAs as a research tool to study gene functions is much more successful than the therapeutic application of small RNAs. This trend will continue at least for the next few years. The therapeutic application of small RNAs is still in its infancy owing to challenges in systemic delivery, off-target effects, and transient gene silencing. Thus, a breakthrough in efficient drug delivery systems is 
indispensable for their future success. However, therapeutic applications of small RNAs for localized diseases are achievable, as these can be administered directly to the disease site, thus avoiding the numerous biological barriers associated with systemic administration.

This theme issue summarizes the recent biological and therapeutic applications of small RNAs. Therapeutic potentials and challenges of small RNAs have been extensively discussed in this theme issue. We hope that this issue will provide a good starting point for current information on small RNAs and stimulate further discussions that may lead to innovative approaches.

\section{What are the challenges for biological and therapeutic applications of small RNAs, and how can be overcome?}

Cheng \& Mahato: Lack of efficient delivery systems is the most critical challenge for the therapeutic applications of small RNAs. While many laboratories and companies are working very hard to develop such delivery systems, the progress is moderate, and a breakthrough is required to boost this field. One problem is that all these laboratories and companies are working independently, trying to develop their own delivery systems. Although many of their "successful" findings have been published, a large number of "failed" results cannot be published and shared with the scientific community, leading to some unnecessary repeats of those "failed" strategies by other scientists. Therefore, we need an agency to gather the experts in this field, summarize what we have learned so far, identify the critical problems, and assign them to the appropriate laboratories. Scientists need to work together, not independently, to solve this biggest challenge that has limited the therapeutic application of small RNAs for a long time. On the other hand, scientific journals should allow scientists to publish "failed" ideas and strategies in emerging and challenging fields. To some extent, these publications may be more valuable to advance this field in the long run.

\section{REFERENCES}

1. Ambros V. The functions of animal microRNAs. Nature. 2004;431 (7006):350-5.

2. Nilsen TW. Mechanisms of microRNA-mediated gene regulation in animal cells. Trends Genet: TIG. 2007;23(5):243-9.

3. Cheng K, Mahato RI. siRNA delivery and targeting. Mol Pharm. 2009;6(3):649-50.

4. Krutzfeldt J, Poy MN, Stoffel M. Strategies to determine the biological function of microRNAs. Nat Genet. 2006;38(Suppl): S14-9.

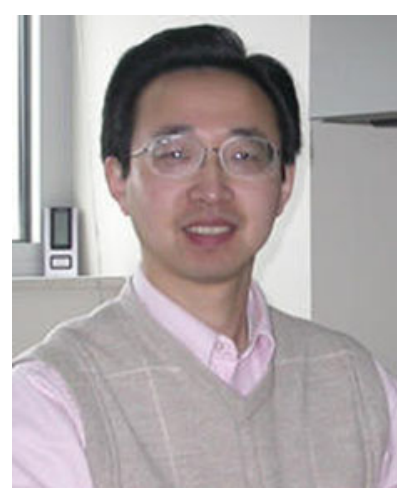

Kun Gheng is an Assistant Professor of Pharmaceutical Sciences at the University of Missouri-Kansas City (UMKG). Dr. Cheng received his B.S. (1996) and M.S. (1999) in Pharmaceutical Sciences from China Pharmaceutical University. He also received another M.S. (2001) in Pharmacy from the National University of Singapore. He worked at the Brightfuture Pharmaceutical Company in HongKong prior to joining the University of Tennessee Health Science Center in 2002, where he received his Ph.D. (2007) in Pharmaceutical Sciences. Dr. Cheng's current research focuses on the development of novel drug delivery systems for small-molecule and macromolecular drugs; identification of tumor-specific artificial ligands for targeted drug delivery and tumor imaging; development of peptide modified prodrugs for chemotherapy; and therapeutic applications of siRNAs for the treatment of breast cancer, prostate cancer, and liver diseases. Much of the effort from Dr. Cheng's laboratory has dealt with the exploration of macromolecular therapeutics which have poor stability and inefficient cellular uptake. His research has been supported by the Department of Defense (DoD) and the National Institute of Health (NIH). Dr. Cheng received a Concept Award from the DoD Breast Cancer Research Program in 2008.

Dr. Cheng is actively involved in various extramural professional activities. He edited a theme issue entitled "siRNA Delivery" for the journal Molecular Pharmaceutics in 2009. He currently serves on the editorial boards of ISRN Pharmaceutics and the International Journal of Pharmaceutical Engineering (IJPHE). Dr. Cheng has also been actively involved in the American Association of Pharmaceutical Scientists (AAPS) UMKG Student Chapter and the Pharmaceutical Sciences Graduate Student Association (PSGSA), serving as the faculty advisor. Dr. Cheng is the recipient of the 2011 AAPS New Investigator Grant Award in Pharmaceutics and Pharmaceutical Technology.

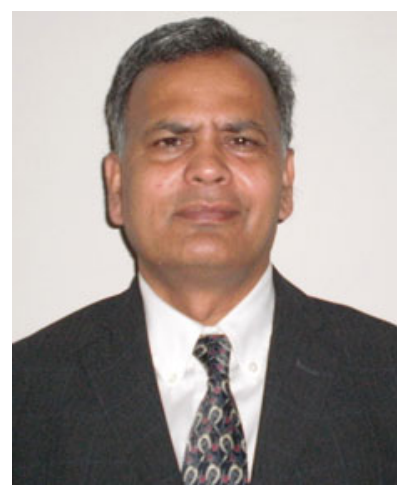

Ram I. Mahato is a Professor of Pharmaceutical Sciences and Biomedical Engineering at the University of Tennessee Health Science Center, Memphis. He was a Research Assistant Professor at the University of Utah, Senior Scientist at GeneMedicine, Inc., and postdoctoral fellow at the University of Southern California in Los Angeles, Washington University in St. Louis, and Kyoto University, Japan. He received a Ph.D. in Drug Delivery from the University of Strathclyde, UK, and B.S. from China Pharmaceutical University, Nanjing. He has published 90 papers, two U.S. patents, six books, and seven journal issues. He is a Special Features Editor of Pharmaceutical Research and editorial board member of eight journals. He is a CRS Fellow (2011), AAPS Fellow (2010), Permanent Member of BTSS/NIH Study section (2009-present), and ASGCT Scientific Advisor (nonviral vectors, 2006-2009). He teaches Pharmaceutical Principles to PharmD students and Drug Delivery and Biocompatibility to graduate students. In 2009, he was honored by the Excellence in Teaching Award from the University of Tennessee. 